\title{
Experimental study and modelling of the kinetics of drying of urban wastewater treatment plant sludge
}

\author{
H. Amadou ${ }^{1}$, J.-B. Poulet ${ }^{2}$, C. Beck ${ }^{1} \&$ A.-G. Sadowski ${ }^{1}$ \\ ${ }^{1}$ ENGEES UPR SHU, Strasbourg, France \\ ${ }^{2}$ INSA, LRIT-ERESA, Strasbourg, France
}

\begin{abstract}
The treatment of sludge by solar drying under a greenhouse is a technique increasingly used for small and medium sized wastewater treatment plants. In order to contribute to the improvement of dimensioning and the effectiveness of the drying of sludge, we propose using the DVS system (Dynamic System of Vapor Sorption), to determine experimentally the isotherms of desorption at 30 and $50^{\circ} \mathrm{C}$. These curves were modelled following a semi-empirical correlation suggested by Oswin. Consequently, experimental results of drying by the forced convection of sludge were obtained, using well controlled thermal conditions of air $\left(30\right.$ and $50^{\circ} \mathrm{C}$ for the temperatures 40 and $60 \%$ for relative humidity 1.0 and $2.0 \mathrm{~m} / \mathrm{s}$ for air velocity). A characteristic curve of drying is built for the different aerothermic conditions. This simplified method makes it possible to simulate the experimental kinetics satisfactorily.
\end{abstract}

Keywords: modelling, isotherm of sorption, kinetics of drying, sludge, solar drying, wastewater treatment.

\section{Introduction}

The treatment of domestic wastewater produces large quantities of residual sludge. Nowadays, wide reduction of these waste volumes is necessary to satisfy new environmental regulations [1].

Sun drying whilst covered is a technique increasingly used for small and medium sized wastewater treatment plants. The control of the solar process of drying of sludge is thus essential. Within this context, the present work 
attempted to assess the performances of this type of process in dewatering and reducing the volume of sludge.

The important stage in every drying process of a material consists in evaluating the hygroscopic character of the product, which is materialized by its curve of sorption. The knowledge of this curve makes it possible to draw from useful information, in particular during the drying and during the storage of sludge.

The complexity of the phenomena intervening during drying, the difficulty in determining certain parameters made certain authors [2-4] directs themselves towards an empirical step.

This step consists in starting from the representation of the results of various experiments carried out under various conditions of temperatures of relative humidity and velocity of air, to determine single curve known as characteristic curve of drying. The concept of characteristic curve of drying seems a method adapted to describe the behavior of sludge.

We initially propose to determine the isotherm of sorption in experiments and to choose a semi-empirical model among those existing in the literature. And in the second time to seek, on the basis of the experimental approach, a semiempirical correlation of the characteristic curve of drying allowing to better simulate the kinetics of drying under various atmospheric conditions.

\section{Materials and methods}

\subsection{Isotherms of desorption}

\subsubsection{Origin and nature of the sludge}

The Studied sludge came from Rosheim urban wastewater treatment plant (11000 population Equivalent) which characteristic was the use of biological treatment process.

The sludge is taken at the end of the mechanical dewatering stage with siccity between 16 and $19 \%$.

\subsubsection{Isotherm desorption methodology}

This technique allows one to describe the changes of the moisture content within the sample in relation to thermodynamic activity of water, at a fixed temperature [5-8].

Experimentally, samples of sludge are placed in containers. The containers are set inside a chamber under controlled humidity and temperature.

The samples are weighed at the thermodynamic equilibrium (which is reached when the weight remains constant over time). At the end of experiment the sludge average moisture content is determined by drying at $105^{\circ} \mathrm{C}$ for $24 \mathrm{~h}$.

The curve representing the evolution of the content of a product according to the relative humidity $\mathrm{Hr}$ of the air for a given temperature $\mathrm{T}$ is called: isotherm of desorption if it was given by a product saturated with water.

The knowledge of the isotherm of desorption makes it possible to calculate the water content at equilibrium of the product which is the limit towards which 
the water content will tighten at the end of drying. The value is a parameter, which appears in particular in the models making it possible to envisage the evolution of the water content of a product during its drying [7].

\subsubsection{Experimental device}

The isotherms of sorption were obtained using DVS system (Dynamic System of Vapor Sorption) of the laboratory of process engineering of the ENSTIMAC. The DVS system has a microbalance with supersensitive continuous recording able to measure changes of mass of the sample lower than 1 part by 10 billion.

This type of microbalance has as principal characteristic to have a very good long-term stability and is consequently ideal for the measurement of phenomena of vapor sorption, which lasts between a few minutes and a few days. This microbalance is placed in an incubator, which makes it possible to generate a temperature of constant and precise measurement.

The required relative humidities are generated by the mixture of a dry steam flow with a saturated steam flow in proportions that are measured with a precision fluxmeter. Combined probes of moisture and temperature are located just below the nacelles of sample and reference in order to allow a checking independent of the performances of the system. The mechanism of the microbalance is very sensitive to the sorption and the desorption of moisture. DVS instrument is completely automated and controlled by computer.

\subsection{Kinetics of drying}

According to drying literature $[4,10]$ the drying curve describes the evolution of evaporation flux versus the mean moisture content. It classically shows tree different phases: a short period of increasing temperature, a period of constant rate, and a period of decreasing drying rate that concludes with the stabilisation of water content at an equilibrium value at the end of drying.

\subsubsection{Experimental unit}

The drying experiments were carried out in a climatic chamber. This experimental device makes it possible to have a controlled aerothermic condition that can be varied during experiments.

The air flow conditions (temperature, relative humidity and velocity) were kept constant for each experiment. The mass and the temperature of sample were recorded on a computer.

The whole of the device comprises:

- The climatic box of section $180 \mathrm{~cm} \times 100 \mathrm{~cm}$ and height $109 \mathrm{~cm}$.

- A support of surface $32 \mathrm{~cm}$ X $26 \mathrm{~cm}$ introduced into the climatic box and posed on an electronic balance of precision $0,01 \mathrm{~g}$, provided with an analogical exit.

- An adjustable ventilator of flow thanks to a variable generator. The velocity of air is measured by two thermal anemometers of type FVA645TH2/TH3 with a precision of $0.01 \mathrm{~m} / \mathrm{s}$. One of them is placed at the upstream of the support and the other at the downstream. 
- A thermo-hygrometrical probe of measurement of type MT8636HR of precision $2 \%$ measuring the temperature and the moisture of the air circulating in the climatic box in order to check the temperature and the hygroscopy of instruction fixed at the level of the climatic chamber.

- A thermocouple of the type K to large standard handle T 150 placed at some depth of sludge. This thermocouple makes it possible to follow the change of the temperature of sludge during the test.

- A station of acquisition of the data connected to a microcomputer equipped with software Amr-CONTROL allowing to store and treat the data.

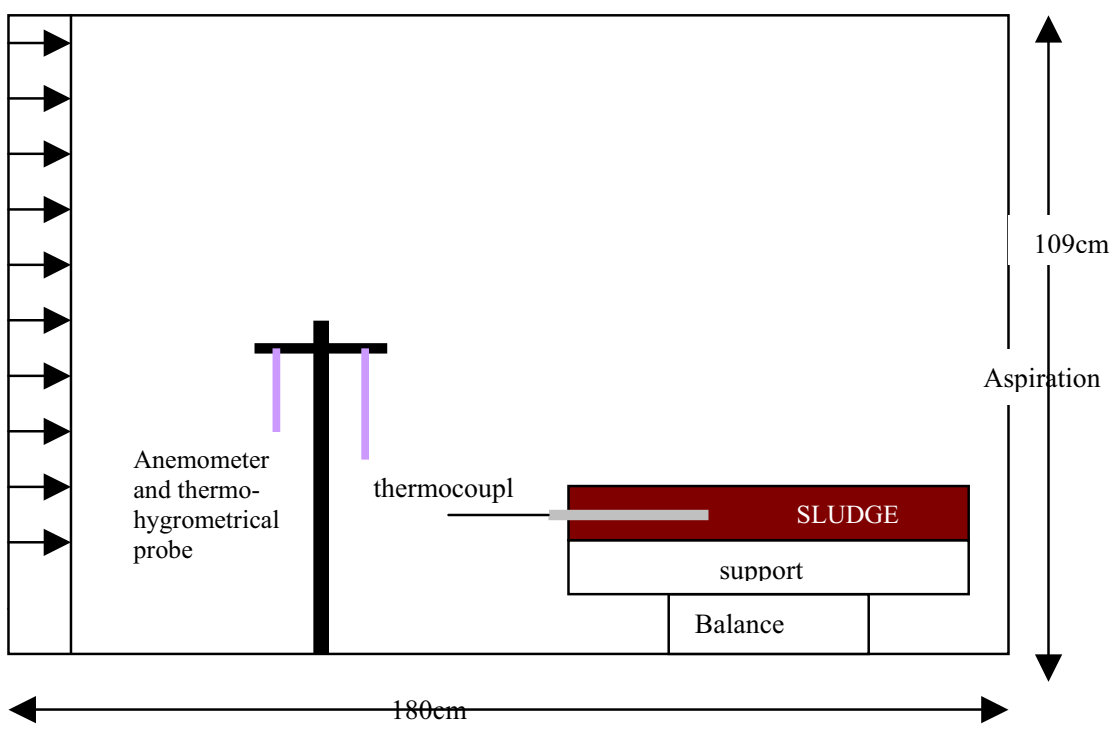

Figure 1: Climatic box.

\subsubsection{Experimental protocol}

The principle of the tests is simple. One fixes the air flow conditions (temperature, relative humidity and velocity), after the system be stabilized, Then one introduces into the climatic box the sludge laid out on the support in thin layer. The mass and the temperature of sample are recorded on a computer. A set of eight tests is envisaged at two temperatures $\left(30\right.$, and $\left.50^{\circ} \mathrm{C}\right)$, two air velocities $(1.0$ and $2.0 \mathrm{~m} / \mathrm{s})$ and at two relative humidities (40 and $60 \%)$.

\section{Experimental results}

\subsection{Experimental results of desorption isotherm}

The desorption isotherm were established at $30^{\circ} \mathrm{C}$ and $50^{\circ} \mathrm{C}$ from water contents at equilibrium, obtained for different degrees of relative humidity. 


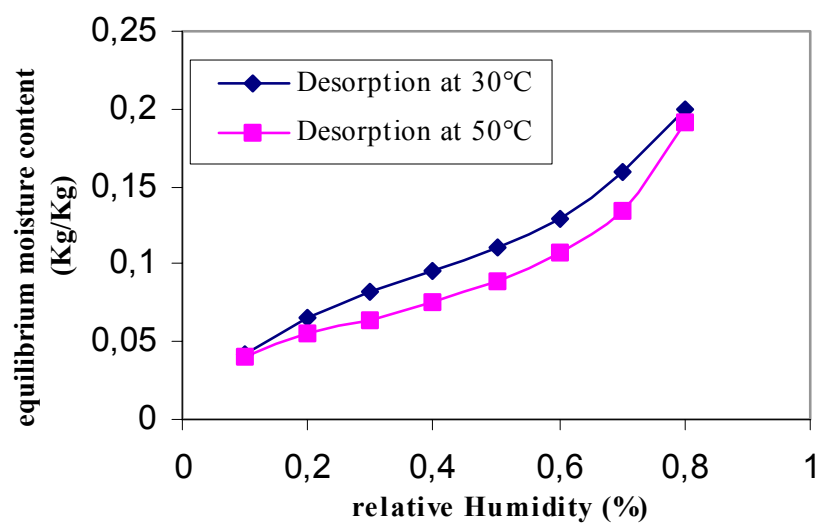

Figure 2: $\quad$ Isotherms of desorption at $30^{\circ} \mathrm{C}$ and $50^{\circ} \mathrm{C}$.

The isotherms obtained were of type II, as is generally the case for urban residual sludge [1]. A large number of models have been proposed in the literature in order to describe the evolution of the equilibrium moisture content with water activity [1,5-8].

A non-linear optimization method was used to fit the parameters of every model and their suitability has been evaluated through the mean relative error (EQM: average standard deviations). According to the EQM criterion the Oswin model best fits the experimental data [3].

\section{Oswin model}

$$
X_{e q}=k\left(\frac{H r}{1-H r}\right)^{n}
$$

$k, n$ are the coefficients characteristic of the product.

Table 1: Parameters of the model of Oswin and EQM relating to the adjustment.

\begin{tabular}{|lllll|}
\hline temperature & Parameter $\mathrm{k}$ & Parameter $\mathrm{n}$ & Field of validity & EQM \\
\hline $30^{\circ} \mathrm{C}$ & 0.112 & 0,416 & $0.10 \leq \mathrm{Hr} \geq 0.80$ & $3.50652 \mathrm{E}-05$ \\
\hline $50^{\circ} \mathrm{C}$ & 0.0938 & 0.484 & $0.10 \leq \mathrm{Hr} \geq 0.80$ & $2.90608 \mathrm{E}-04$ \\
\hline
\end{tabular}

These curves indicate a relatively good agreement between the model and the experimental data. 


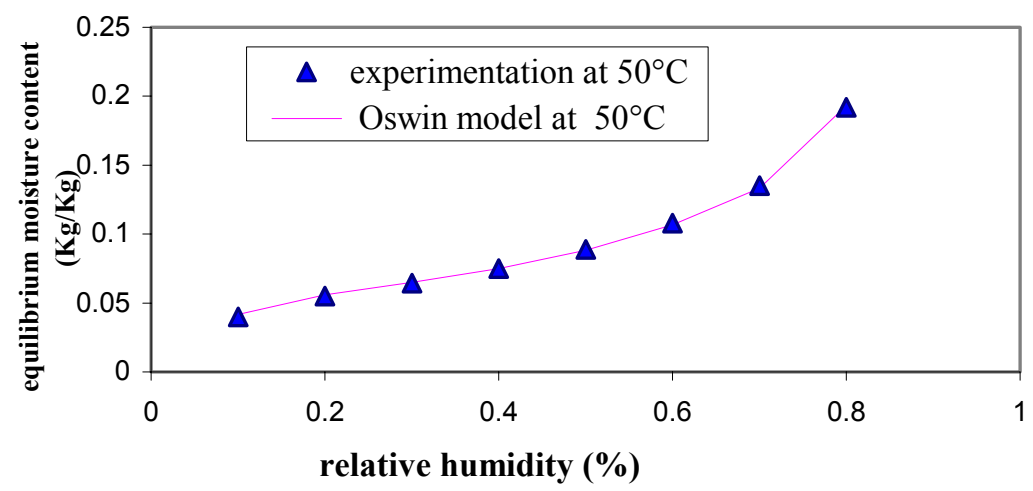

Figure 3: $\quad$ Model/experimentation at $50^{\circ} \mathrm{C}$.

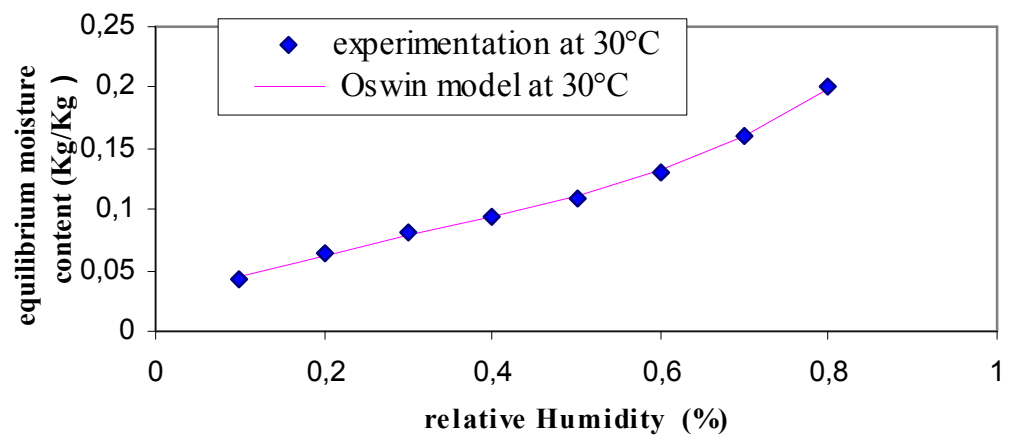

Figure 4: $\quad$ Model /experimentation at $30^{\circ} \mathrm{C}$.

\subsection{Experimental results of drying kinetics}

\subsubsection{Curves of drying}

The curves of drying indicate, either the variations of the moisture content of sludge according to time, or that giving drying rate according to time, or even the curve proposed by Krischer and Kröll [4] drying rate according to the moisture content of sludge, or finally the reduced shape of the curve of krischer for a single representation of various conditions of drying.

The curve of drying rate can be obtained, by calculating the derivative after smoothing of the curve or directly starting from the experimental points.

\subsubsection{Identification of the curve characteristic of drying}

The representation of the results of various experiments for a given product, proposed by Van Meel then Krischer and Schlunder [1,2,3], makes it possible to plot the characteristic curve of drying. This curve makes possible the regrouping of the results achieved under different conditions of air (temperature, relative humidity, and velocity). The kinetics of drying are represented in the form: 


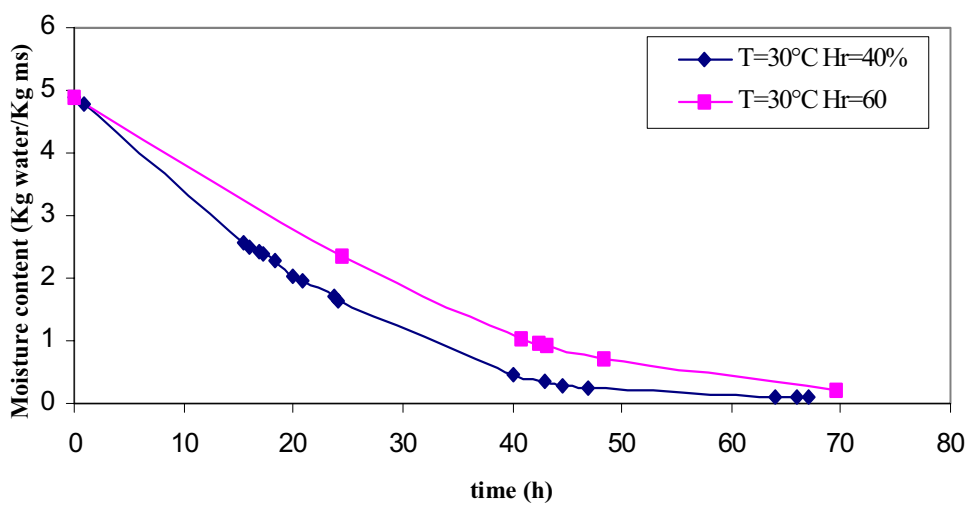

Figure 5: $\quad$ Moisture content for two conditions air for sludge.

$$
\left(-\frac{d X}{d t}\right)=\left(-\frac{d X}{d t}\right)_{1} f\left(X_{r}\right)
$$

$X_{r}$ is the reduced moisture content defined by:

$$
X_{r}=\frac{X-X_{e q}}{X_{c r}-X_{e q}}
$$

$X_{e q}$ is the equilibrium water content measured after drying

$\left(-\frac{d X}{d t}\right)_{1}$ is the constant rate period $X_{i} \leq X \leq X_{c r}$

$f\left(X_{r}\right)$ is the normalized drying rate

Generally, the characteristic curves are represented by functions of the polynomial type or power type. In this framework we will use the polynomial function presented in the following form:

$$
f\left(X_{r}\right)=A_{1} X_{r}+A_{2} X_{r}^{2}+A_{3} X_{r}^{3}
$$

The constants, $A_{1}, A_{2}, A_{3}$ are given in the experiments for fixed characteristics of air.

\subsubsection{Determination of the first phase and the moisture content critical} The constant rate period is not easily identifiable, even for the products whose initial moisture is important. The explanation lies in the fact that the cellular walls disturb the fast migration of moisture towards the surface of the products [5]. Thus several authors [11,12] identify the moisture content critical with the initial content.

The constant rate period can be given by using a correlation, which includes the external aerodynamic conditions, and by taking in to account the deformable 
character of the biological products or by using the analogy of the transfers of heat and mass through the boundary layer of a completely wet surface. This phase is comparable with the evaporation of free water. The temperature of the solid remains constant and equal to the wet bulb temperature. The constant rate ( $\mathrm{Kg}$ of water /s) is thus equal to:

$$
m_{s}\left(\frac{d X}{d t}\right)_{1}=\frac{h S}{L_{v}}\left(T_{a}-T_{b}\right)
$$

\section{Perspective}

At the end of our experimental tests, we will represent the profiles of the kinetics of drying for various values of temperature, relative humidity and velocity of air. Those profiles, will allow us:

- $\quad$ To identify the parameters, $A_{1}, A_{2}, A_{3}$, for each test

- To determine, the average values of $A_{1}, A_{2}, A_{3}$ and the standard deviations of these parameters for the whole of the experimental tests.

Some tests, apart from those, which made it possible to fix the curve, will allow us to validate the obtained model. These experimental tests will also allow us to show the influence of the characteristics of the air on the speed of drying.

\section{Conclusion}

The isotherms of desorption of sludge were obtained by using DVS system (dynamic System of vapor sorption). The obtained results were simulated by the semi-empirical model of OSWIN.

Then, the influence of drying air velocity, drying air temperature and relative humidity on the drying kinetics of sludge will be studied. The characteristic curve of drying and the expression of drying rate will be elaborated from the experimental results. This formula is necessary for programs simulating solar drying systems.

\section{Nomenclature}

$A_{1}, A_{2}, A_{3} \quad$ Coefficients characteristic of drying.

$\mathrm{Hr}$ Relative humidity of the air

$h \quad$ Convection coefficient..................................................... $\left(\mathrm{W} / \mathrm{m}^{2} / \mathrm{K}\right)$

$k, n$ Coefficients characteristic of the product

$l_{v} \quad$ Latent heat of water vaporization.

$m_{b}$ Mass of sludge

$m_{s}$ Mass Dries 


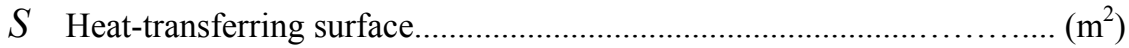

$T_{a}$ Temperature of the air drying.......................................................... $\left({ }^{\circ} \mathrm{C}\right)$

$T_{b}$ Temperature of sludge............................................................... $\left({ }^{\circ} \mathrm{C}\right)$

$t$ Time

$\mathrm{U}$ Air velocity

$X$ Moisture content dry basis.

.(Kg water/kg dry solid)

$\left(\frac{d X}{d t}\right)_{1}$ Speed of drying with constant phase

(Kg water/kg dry solid.h)

\section{Subscripts}

$\begin{array}{ll}b & \text { Sludge } \\ c r & \text { Transition moisture content: bound-free water } \\ e q & \text { At thermodynamic equilibrium } \\ i & \text { Initial }\end{array}$

\section{References}

[1] Vaxelaire J, Mousques P, Bongiovanni J.M and Puiggali J.R., Desorption of domestic activated sludge Environmental Technology, vol. 21 pp 327335 (2000).

[2] Van Meel (D.A.). - Chem. Eng. Sci., 9, p. 36 (1957).

[3] Schlunder (E.V.). - Handbook of heat transfer. Section 3: Dryers, Hemisphere Publishing Corp, (1983).

[4] Krischer (O.) et Kröll (K.). - Technique du séchage. cetiat Orsay, 599 p.Traduction de Die Wissenschaftlichen Grundlagen der Trocknungstechnik. Springer Verlag Berlin (1963).

[5] Vaxelaire J., Bongiovanni J.M and Puiggali J.R., Mechanical dewatering and thermal drying of residual sludge. Environnemental Technology, vol 20 pp 29-36 (1999).

[6] Léonard A., Etude du séchage convectif de boues de station d'épuration, suivi de la texture par microtomographie à rayons $\mathrm{x}, \mathrm{PhD}$ thesis, University of Liège (2003).

[7] Nelson R.M., A model for sorption of water by cellulosic materials. Wood and Fiber Science, 15,8-22 (1983).

[8] Talla A, Jannot Y, Kaspseu C, Nganhou J., Experimental study and modelling of kinetics of drying of tropical fruits. Application to banana and to mango. Wood science, 21 499-518 (2001).

[9] Lahsasni S, Kouhila M, Mahrouz M., Experimental study of drying kinetics of cladode of opuntia ficus indica. International forum on Renewable Energies FIER 254-259 (2002).

[10] Charreau A, Cavaillé R., Séchage: Théorie et calculs. Techniques de l'ingénieur, traité Génie des procédés, J 2 480-1. 
142 Advances in Fluid Mechanics VI

[11] Desomorieux H., Moyne C., Analysis of dryers performance for tropical foodstuffs using the characteristic drying curve concept. In: Drying 92, Elsevier Amsterdam, 834-843 (1992).

[12] Formell A., Séchage de produits biologiques par l'air chaud: calcul de séchoirs. PhD thesis, Montpellier (1979). 